\title{
Incidence and Predictive Factors of Distal Pedicle Screw Loosening Followed Posterior Corrective Surgery for Degenerative Lumbar Scoliosis
}

Hui Wang

Peking University Third Hospital

Longjie Wang

Peking University Third Hospital

Zhuoran Sun

Peking University Third Hospital

Shuai Jiang

Peking University Third Hospital

Weishi Li (D puh3liweishi@163.com )

Peking University Third Hospital

Research article

Keywords: distal pedicle screw loosening, posterior surgery, degenerative lumbar scoliosis

Posted Date: January 25th, 2021

DOI: https://doi.org/10.21203/rs.3.rs-150236/v1

License: (c) (1) This work is licensed under a Creative Commons Attribution 4.0 International License.

Read Full License 


\section{Abstract}

\section{Background}

The aim of this study was to identify the prevalence of distal pedicle screw loosening (DPSL) following posterior decompression and instrumented fusion for DLS, to search for predictable risk factors for DPSL, and hope to provide references in decision making and surgical planning for spine surgeons.

\section{Methods}

One hundred and fifty-three consecutive DLS patients surgically treated between January 2013 and July 2018 were retrospectively reviewed. The diagnostic criteria for DPSL developed by X-ray include the radiolucent area (thicker than $1 \mathrm{~mm}$ ) around screw and the "double halo" sign defined as the presence of radiolucent area and radiopaque rim at the same X-ray. According to the occurrence of DPSL at two-year follow-up, patients were divided into two groups: study group (screw loosening) and control group (without screw loosening). To investigate predictive factors for distal pedicle screw loosening, three categorized factors were analyzed statistically.

\section{Results}

DPSL was detected in 72 of 153 patients (47.1\%) at two-year follow up, and were enrolled as study group. Another 81 patients presented no screw loosening at two-year follow up, and were enrolled as control group. Hounsfield unit (HU) value was lower in study group than that in control group. Fusion level was longer in study group than that in control group. Lower instrumented vertebrae on L5 was less in study group than that in control group. Posterolateral fusion was less in study group than that in control group. Preoperative Cobb angle, postoperative Cobb angle, Cobb angle correction, preoperative lumbosacral coronal angle (LSCA), LSCA correction, preoperative thoracolumbar junction (TL), postoperative TL were larger in study group than those in control group. Logistic regression analysis revealed that low Bone mineral density (BMD) (<169 HU), posterolateral fusion, Cobb angle correction ( $>16$ degrees), LSCA correction (>9 degrees) were independently associated with DPSL.

Conclusions

The incidence of DPSL following posterior decompression and instrumented fusion for DLS is $47.1 \%$. Low BMD, large correction of both main curve and fractional curve are predictive factors for DPSL, while posterolateral fusion is a protective factor.

\section{Background}

Degenerative lumbar scoliosis (DLS) is characterized as three-dimensional deformity, presenting not only scoliotic deformity on coronal plane, but also lumbar hypo-lordosis on sagittal plane [1]. Posterior decompression and instrumented fusion is supposed to be the most commonly used surgical procedure for DLS [2]. Scoliosis correction and lumbar lordosis reconstruction could be achieved by pedicle screw 
compression and distraction [3]. Screws loosening is one of the frequently reported typical complication of pedicle screws fixation, and usually occurs in the cranial and caudal vertebrae of the long fusion, much more frequent in caudal screws [4]. Screw loosening may lead to pseudarthrosis, nonunion, loss of correction, revision surgery, and therefore need to be paid specific attention [5]. The frequency of screw loosening has varied widely in different studies, which ranged from less than $1 \%$ to $15 \%$ in nonosteoporotic patients and even up to $60 \%$ in osteoporotic spines, depending on the study population and the duration of follow-up [6-8]. Knowledge of risk factors is very important for minimizing the occurrence of screw loosening and to allow surgeons to take measures for its prevention when possible.

Bone mineral density (BMD) is supposed to be an important factor influencing the stability of pedicle screws [9]. Wittenberg et al. proved that early loosening of pedicle screws may be expected at a BMD less than $90 \mathrm{mg} / \mathrm{mL}$, as measured by quantitative computed tomography [10]. The majority of studies were focused on impact of BMD on the occurrence of screws loosening, however, it has been proven that bone quality is not the only contributing factor $[8,11,12]$. Several other factors affecting the stability of pedicle screws, such as the length, outer diameter, design, fitness in the pedicle, elasticity of the cancellous bone, were supposed to potentially influence the development of screw loosening [13-15]. Although previous reports have alerted spine surgeons about screw loosening subsequent to posterior decompression and instrumented fusion, very few published reports describing specific risk factors that need to be taken into account to avoid this undesirable event for DLS patients.

The aim of this study was therefore to identify the prevalence of distal pedicle screw loosening (DPSL) following posterior decompression and instrumented fusion for DLS, to search for predictable risk factors for DPSL, and hope to provide references in decision making and surgical planning for spine surgeons.

\section{Methods}

\section{Patients}

A retrospective study was conducted, it was approved by the Institutional Review Board of Peking University Third Hospital before data collection and analysis. One hundred and fifty-three consecutive DLS patients surgically treated between January 2013 and July 2018 were retrospectively reviewed. Inclusion criteria: 1. DLS patients treated by posterior instrumented fusion with pedicle screws. 2 . followup duration more than two years with radiological data including antero-posterior $(A / P)$ and lateral X-ray at preoperation, early postoperation, and two-year follow-up. Exclusion criteria: 1. patients with spinal deformities derived from idiopathic scoliosis, ankylosing spondylitis, neuromuscular diseases, infections, or scheuermann kyphosis. 2. patients treated with anterior or lateral lumbar fusion surgery, minimally invasive lumbar fusion surgery. 3. posterior instrumentation include iliac screw (IS) or sacral-alar-iliac (S2Al).

\section{Clinical and radiological evaluation}


The diagnostic criteria for DPSL developed by X-ray include the radiolucent area (thicker than $1 \mathrm{~mm}$ ) around screw and the "double halo" sign defined as the presence of radiolucent area and radiopaque rim at the same X-ray [16,17]. (figure 1) The first three authors resolved all ambiguities by consensus. According to the occurrence of DPSL at two-year follow-up, patients were divided into two groups: study group (screw loosening) and control group (without screw loosening). To investigate predictive factors for distal pedicle screw loosening, three categorized factors were analyzed statistically.

Patient general data including age, sex, body mass index (BMI), BMD, comorbidity (hypertension, diabetes, coronary heart disease) were investigated. BMD was evaluated by Hounsfield unit (HU) value on L1 from computed tomography (CT), which was performed via a previously described technique using Philips Easy Vision Picture Archiving and Communication System (PACS) [18]. This protocol records HU on axial images at three levels within the vertebral body: immediately caudal to the superior end plate, mid-body, and cranial to the inferior end plate. (figure 2) For each measurement, the largest possible elliptical region of interest was drawn, excluding the cortical margins to prevent volume averaging.

Surgical data including surgery time, estimated blood loss, fusion level, lower instrumented vertebrae (LIV), Posterolateral fusion were reviewed.

Radiological data were collected and evaluated at pre-operation, early post-operation, two years postoperation. Cobb's angle was measured using lines projected from the upper border of the most tilted vertebra above and lower border of the most tilted vertebra within the main curve. Lumbosacral coronal angle (LSCA) was measured using lines projected from the upper border of L4 and upper border of S1 on $\mathrm{P} / \mathrm{A}$ X-ray. Coronal balance distance (CBD) was measured as the distance between $\mathrm{C7}$ plumb line and center sacral vertical line. Lumbar lordosis (LL) was measured from T12 inferior endplate to S1 superior endplate by the Cobb method on lateral X-ray. Thoracolumbar junction (TL) was measured from T10 superior endplate to L2 inferior endplate on lateral X-ray. Pelvic incidence $(\mathrm{PI})$ was defined as the angle between the line perpendicular to the sacral plate and the line connecting the midpoint of the sacral plate to the bicoxofemoral axis. Sacral slope (SS) was the angle between the S1 superior endplate and a horizontal line. Sagittal vertical axis (SVA) was the distance from C7 plumb line to the perpendicular line drawn from superior posterior endplate of S1. (figure 3)

\section{Statistical analysis}

Data were analyzed using Statistical Product and Service Solutions software (version 17; SPSS, Chicago, IL). Continuous variables were measured as mean \pm standard deviation, and categorical variables were expressed as frequency or percentages. An independent $t$ test was used to analyze the difference of continuous variables between two groups. An $\chi 2$ analysis and Fisher's exact test were used to examine the differences among categorical variables. Binary Logistic regression analysis was used to analyze the assumed predictive factors with backward elimination, in which variables with a significance level of $>.10$ were removed. The confidence interval of the odds ratio (OR) was $95 \%$. 


\section{Results}

DPSL was detected in 72 of 153 patients (47.1\%) at two-year follow up, and were enrolled as study group. Another 81 patients presented no screw loosening at two-year follow up, and were enrolled as control group. (figure 4 and 5)

There was no statistically significant difference between the two groups in age at operation, sex, BMI, comorbidity. The HU value on L1 was lower in study group than that in control group. (Table 1)

There was no statistically significant difference between the two groups in mean surgery time, estimated blood loss. Fusion level was longer in study group than that in control group. The LIV on L5 was less in study group than that in control group. Posterolateral fusion was less in study group than that in control group. (Table 2)

The preoperative Cobb angle, postoperative Cobb angle, correction of Cobb angle, preoperative LSCA, correction of LSCA, preoperative TL, postoperative TL were larger in study group than those in control group. There was no statistically significant difference in postoperative LSCA, preoperative CBD, postoperative $C B D$, correction of $C B D$, preoperative $L L$, postoperative $L L$, correction of $L L$, correction of $T L$, $\mathrm{PI}$, preoperative SS, postoperative SS, correction of SS, preoperative SVA, postoperative SVA, correction of SVA between the two groups. (Table 3)

The following variables were entered into the Logistic regression model: BMD (HU value on L1), fusion level, LIV, posterolateral fusion, preoperative Cobb angle, postoperative Cobb angle, correction of Cobb angle, preoperative LSCA, correction of LSCA, preoperative TL, postoperative TL. Binary Logistic regression model revealed that BMD (HU value on L1), posterolateral fusion, correction of Cobb angle, LSCA correction were independently associated with DPSL. (Table 4) 
Table 1 Comparison of general data between study and control groups

\begin{tabular}{|lllll|}
\hline & Study group & Control group & statistic & $p$ value \\
\hline Number of cases & 72 & 81 & - & - \\
\hline Age & $62.8 \pm 6.6$ & $64.7 \pm 6.7$ & -1.657 & 0.100 \\
\hline Sex (M/F) & $17 / 55$ & $21 / 60$ & 0.109 & 0.741 \\
\hline BMI & $25.7 \pm 3.3$ & $26.7 \pm 3.4$ & -1.119 & 0.268 \\
\hline BMD (HU value on L1) & $135.8 \pm 57.8$ & $198.9 \pm 66.6$ & -6.222 & $<0.001$ \\
\hline Comorbidity & & & & \\
\hline Hypertension (yes/no) & $27 / 45$ & $32 / 49$ & 0.065 & 0.799 \\
\hline Diabetes (yes/no) & $18 / 54$ & $19 / 62$ & 0.050 & 0.824 \\
\hline Coronary heart disease (yes/no) & $9 / 63$ & $11 / 70$ & 0.039 & 0.843 \\
\hline Mean values are presented as \pm standard deviation. & & \\
\hline BMl indicates body mass index; BMD indicates bone mineral density; HU indicates Hounsfield unit. \\
\hline
\end{tabular}

Table 2

Comparison of surgical data between study and control groups

\begin{tabular}{|lllll|}
\hline & Study group & Control group & statistic & $p$ value \\
\hline Surgery time (min) & $226.2 \pm 37.6$ & $215.5 \pm 83.9$ & 0.638 & 0.527 \\
\hline Estimated blood loss $(\mathrm{mL})$ & $792.5 \pm 371.5$ & $711.2 \pm 386.5$ & 0.805 & 0.424 \\
\hline Fusion level & $6.8 \pm 1.8$ & $5.5 \pm 1.5$ & 4.940 & $<0.001$ \\
\hline LIV (L5/S1) & $25 / 47$ & $45 / 36$ & 6.666 & 0.010 \\
\hline Posterolateral fusion (yes/no) & $4 / 68$ & $43 / 38$ & 40.463 & $<0.001$ \\
\hline Mean values are presented as \pm standard deviation. & & \\
\hline LIV indicates lower instrumented vertebrae. & & \\
\hline
\end{tabular}


Table 3

Comparison of peri-operative radiological data between study and control groups Study group

Control group

statistic

$p$ value

Cobb angle

Preoperative value

$32.1 \pm 11.1$

$25.3 \pm 11.6$

3.618

$<0.001$

Postoperative value

$12.8 \pm 7.4$

$8.9 \pm 6.3$

2.837

0.005

Correction

$21.9 \pm 10.9$

$17.6 \pm 10.8$

2.440

0.016

LSCA

LSCA

Preoperative value

$17.7 \pm 5.9$

$13.3 \pm 3.8$

5.528

$<0.001$

Postoperative value

$6.7 \pm 3.7$

$6.3 \pm 2.9$

0.745

0.458

Correction

$10.9 \pm 4.4$

$7.0 \pm 2.6$

6.835

$<0.001$

CBD

Preoperative value

$19.4 \pm 13.2$

$23.4 \pm 19.5$

$-1.076$

0.285

Postoperative value

$8.5 \pm 7.3$

$10.4 \pm 7.6$

$-0.810$

0.422

Correction

$7.1 \pm 13.6$

$7.3 \pm 16.2$

$-0.083$

0.934

LL

Preoperative value

$-3.1 \pm 29.7$

$3.0 \pm 30.2$

$-1.227$

0.222

Postoperative value

$10.9 \pm 38.7$

$17.5 \pm 36.4$

$-4.242$

0.427

Correction

$12.5 \pm 21.6$

$15.7 \pm 18.7$

$-1.569$

0.519

TL

Preoperative value

$18.7 \pm 14.7$

$13.5 \pm 12.5$

2.278

0.024

Postoperative value

$16.3 \pm 9.2$

$11.8 \pm 11.3$

2.307

0.023

Correction

$-5.9 \pm 14.8$

$-4.5 \pm 11.1$

$-0.671$

0.503

PI

$46.5 \pm 10.9$

$47.7 \pm 11.6$

$-0.593$

0.554

SS

Preoperative value

$24.7 \pm 9.8$

$24.3 \pm 10.6$

0.019

0.985

Mean values are presented as \pm standard deviation.

LSCA indicates lumbosacral coronal angle; CBD indicates coronal balance distance; $L L$ indicates lumbar lordosis; TL indicates thoracolumbar lordosis; PI indicates pelvic incidence; SS indicates sacral slope; SVA indicates sagittal vertical axis. 


\begin{tabular}{|lllll|}
\hline & Study group & Control group & statistic & p value \\
\hline Postoperative value & $28.7 \pm 8.0$ & $30.1 \pm 8.1$ & -0.899 & 0.370 \\
\hline Correction & $-0.4 \pm 14.4$ & $-1.3 \pm 14.8$ & 0.344 & 0.731 \\
\hline SVA $(\mathrm{mm})$ & & & 1.582 & 0.116 \\
\hline Preoperative value & $50.6 \pm 57.1$ & $37.9 \pm 39.4$ & 1.880 & 0.064 \\
\hline Postoperative value & $28.4 \pm 28.9$ & $16.2 \pm 30.1$ & 0.316 & 0.752 \\
\hline \begin{tabular}{l} 
Correction \\
\hline
\end{tabular} & $30.5 \pm 53.2$ & $28.1 \pm 42.1$ & & \\
\hline $\begin{array}{l}\text { Mean values are presented as } \pm \text { standard deviation. } \\
\text { LSCA indicates lumbosacral coronal angle; CBD indicates coronal balance distance; LL indicates } \\
\text { lumbar lordosis; TL indicates thoracolumbar lordosis; PI indicates pelvic incidence; SS indicates } \\
\text { sacral slope; SVA indicates sagittal vertical axis. }\end{array}$ & & \\
\hline
\end{tabular}

Table 4

Risk factors for pedicle screw loosening, identified by Logistic regression analysis.

\begin{tabular}{|lll|}
\hline Risk factors & Odds ratio [95 \% Cl] & $p$ value \\
\hline BMD (HU value on L1) & $10.672[2.064-55.188]$ & 0.005 \\
\hline Posterolateral fusion & $0.013[0.001-0.126]$ & $<0.001$ \\
\hline Fusion level & $0.223[0.038-1.299]$ & 0.095 \\
\hline Preoperative Cobb angle & $3.504[0.349-35.176]$ & 0.287 \\
\hline Postoperative Cobb angle & $0.158[0.014-1.785]$ & 0.136 \\
\hline Correction of Cobb angle & $7.197[1.028-50.389]$ & 0.047 \\
\hline Preoperative LSCA & $1.708[0.324-9.105]$ & 0.528 \\
\hline LSCA correction & $7.202[1.075-48.260]$ & 0.042 \\
\hline Preoperative TL & $0.262[0.048-1.450]$ & 0.215 \\
\hline Post-operative TL & $2.491[0.226-27.457]$ & 0.456 \\
\hline LIV (L5/S1) & $1.379[0.965-1.971]$ & 0.077 \\
\hline $\begin{array}{l}\text { BMD indicates bone mineral density; HU indicates Hounsfield unit; LSCA indicates lumbosacral } \\
\text { coronal angle; TL indicates thoracolumbar lordosis; LIV indicates lower instrumented vertebrae. }\end{array}$ \\
\hline
\end{tabular}

\section{Discussion}

In the current study, $47.1 \%$ of the patients (72/153) presented DPSL followed posterior decompression and instrumented fusion for DLS, we found that low BMD, large correction of both main curve and fractional curve, posterolateral fusion were significantly and independently associated with the 
occurrence of DPSL, and can be assessed before surgery, these results were not confounded by other variables potentially affect DPSL.

A critical point for the investigation of screw loosening is the assessment of whether a screw is loosened or not, planar radiographs are most commonly used, whereas a significant minority of papers employed clinical CT or low-dose CT [19-21]. Potential health risks associated with increased radiation exposure as well as financial costs limit postoperative CT use to selected patients with persistent symptoms or where there is specific concern regarding the possibility of implant-related complications [5]. Wu et al. proved that CT might not be superior to X-ray in assessment of screw loosening, especially considering its higher cost and radiation exposure, as X-ray had a sensitivity of $24 \%$ and a specificity of $98 \%$, while CT scan had a sensitivity of $22 \%$ and a specificity of $95 \%$ regarding extraction torque as criterion of screw loosening [22]. Sanden et al. reported that a radiolucent zone around a PS is a good indicator of screw loosening [19]. That is the reason why we chose plain radiographs to evaluate pedicle screw loosening in the present study.

Since its first description by Watkins in 1953, the technique of posterolateral fusion (PLF) has evolved into the widely adopted method of lumbar arthrodesis for degenerative disease [23]. Tsirikos et al. proved that posterolateral arthrodesis in situ with autologous iliac crest bone without instrumentation achieved a solid fusion and produced excellent clinical outcomes and high patient satisfaction [24]. Moreover, the use of pedicle screw fixation to support the PLF could achieve fusion rates as high as $96 \%$ [25-27]. Addition of interbody fusion via a transforaminal approach (TLIF) has become a popular surgical option for treatment of degenerative lumbar conditions. Although technically more complicated than PLF, it has been suggested that TLIF provides superior immediate stability and protects against early pedicle screw loosening. Kim et al proved that TLIF appears to have a protective effect in reducing rates of early screw loosening by approximately $60 \%$ versus PLF [28]. A retrospective study comparing the outcomes between TLIF and PLF found that the PLF construct was more prone to induce instrument breakage and pseudoarthrosis compared with the TLIF technique, indicating the importance of anterior column support in the maintenance of construct stability [29]. In recent years, TLIF has replaced PLF to be the most commonly used lumbar fusion surgery [30]. However, postoperative screw loosening was not completely avoided, especially in patients undergoing long segment pedicle screw fixation [5]. Results from the current study suggest that supplementing the TLIF with PLF potentially reduces the rates of DPSL followed corrective surgery for DLS. Following decortication of transverse processes and posterolateral bone, morselized local autograft was placed in the posterolateral intertransverse space in order to create posterolateral arthrodesis, which could reduce the segmental motion of posterior spinal column, and minimize the risk of screw loosening.

It has been widely accepted that osteoporosis is a potential risk factor for pedicle screw loosening. Fixation failure rates due to screw loosening range from $1-15 \%$ and can rise in osteoporotic spines for which the strength of fixation with pedicle screws decreases along with lower bone density [6-8]. In a study of osteoporotic cadavers, pedicle screw pullout strength was found to be highly correlated with BMD [31]. BMD measuring with DEXA scans could not perform differentiation of the cancellous bone 
from the cortical bone, T-scores can be overestimated due to abdominal vessel wall calcification, degenerative bony spurs, and facet hypertrophy $[32,33]$. It has been proved that older patients or patients with degenerative lumbar scoliosis are more likely to have unreliable lumbar T-scores [34]. Measuring HU from computed tomography has been proposed as a useful technique for assessing vertebral bone quality, the reliability and accuracy of HU to determine osteoporosis have been proved in the literature with many reports [35-38]. In the current study, our findings are consistent with Jan et al that the determination of bone density by $\mathrm{HU}$ value could predict the risk of screw loosening and inform the decision to use cement augmentation to reduce the incidence of screw loosening [39]. Screw augmentation with bone cement as well as expandable screws demonstrated improved screw pullout stability, better fixation strength and clinical results in comparison with standard pedicle screws in human cadaver material in vitro [40-43].

The typical major curve of DLS always lies in the mid-lumbar spine, it is always compensated by a distal curve at the lumbosacral region as the body attempts to maintain coronal balance, named the fractional curve $[44,45]$. Both pedicle screws compression at the convex side and pedicle screws distraction at the concave side could achieve correction of the main curve and fractional curve [46, 47]. No matter which technique is used, the cutting effect of pedicle screw on bone should not be ignored. The more scoliosis correction, the greater the cutting effect of pedicle screw on bone, and the greater the risk of pedicle screw loosening. Based on our previous study, we put forward two suggestions. Firstly, asymmetrical vertebral degeneration in DLS patients is manifested as high $\mathrm{HU}$ value within concavity and low $\mathrm{HU}$ value within convexity both in major and fractional curve. Concave side of the vertebrae theoretically withstand more cutting forces than convex side based on the $\mathrm{HU}$ value measurement, distraction of pedicle screws at concave side should be a priority to correct both main curve and fractional curve [48]. Secondly, inadequate release of the posterior spinal bone elements in PLIF or TLIF technique may hinder the correction of the fractional curve, because the lumbosacral junction tends to be particularly rigid and may already be fused into an abnormal position. Posterior column osteotomy facilitate effective correction of the scoliosis through complete release of dural sac as well as the asymmetrical intervertebral reconstruction by cage is recommended [49].

There are several potential limitations in this study. First, the number of patients is relatively small, and the study may be under powered to detect the significance of some risk factors. Second, the study was conducted retrospectively by case selection, and was not randomized and controlled, the subjects selected are all Chinese Han individuals, whether the conclusion is applicable to other ethnic groups needs to be further investigated in the future. Even with these issues, three suggestions are provided for surgical planning based on the findings in the current study. Firstly, screw augmentation with bone cement or expandable screws is recommended for patients with low BMD measured from preoperative CT scan. Secondly, posterior column osteotomy plus unilateral cage strutting technique on the concavity is recommended for scoliosis correction, screw distraction instead of compression should be preferred. Thirdly, posterolateral fusion is recommended for DLS patients that underwent posterior long segment fusion. 


\section{Conclusion}

The incidence of DPSL following posterior decompression and instrumented fusion for DLS is $47.1 \%$. Low BMD, large correction of both main curve and fractional curve are predictive factors for DPSL, posterolateral fusion is a protective factor.

\section{Declarations}

Ethics approval and consent to participate: All procedures performed in studies involving human participants were in accordance with the ethical standards of the institutional and/or national research committee and with the 1964 Helsinki declaration and its later amendments or comparable ethical standards. For this type of study, the informed consent was waived. This study was approved by Peking University Third Hospital Ethics Committee.

Consent for publication: Not applicable

Availability of data: The data used and analyzed during the current study was available from the corresponding author on reasonable request.

Competing interests: The authors declare that they have no competing interests.

Funding: Not applicable

Authors' contributions: WS L conceived the project and analyzed the data. All authors contributed towards the interpretation and the collection of the data. All authors wrote and approved the final manuscript.

Acknowledgements: Not applicable

\section{References}

1. Wong E, Altaf F, Oh LJ, Gray RJ (2017) Adult degenerative lumbar scoliosis. Orthopedics, 40:e930e939. doi: 10.3928/01477447-20170606-02.

2. Wang H, Ma L, Yang D, Wang T, Liu S, Yang S, Ding W (2017) Incidence and risk factors of adjacent segment disease following posterior decompression and instrumented fusion for degenerative lumbar disorders. Medicine (Baltimore), 96:e6032. doi: 10.1097/MD. 0000000000006032.

3. Silva FE, Lenke LG (2010) Adult degenerative scoliosis: evaluation and management. Neurosurg Focus, 28:E1. doi: 10.3171/2010.1.FOCUS09271.

4. Masashi U, Jun T, Shota I, Shugo K, Masayuki S, Toshimasa F, Hiroki O, Michihiko K, Hiroyuki K (2017) Pedicle screw loosening after posterior spinal fusion for adolescent idiopathic scoliosis in upper and lower instrumented vertebrae having major perforation. Spine (Phila Pa 1976), 42:18951900. doi: 10.1097/BRS.0000000000002305. 
5. Galbusera F, Volkheimer D, Reitmaier S, Berger-Roscher N, Kienle A, Wilke HJ (2015) Pedicle screw loosening: a clinically relevant complication? Eur Spine J, 24):1005-1016. doi: 10.1007/s00586-0153768-6.

6. K Okuyama, E Abe, T Suzuki, Y Tamura, M Chiba, K Sato (2000) Can insertional torque predict screw loosening and related failures? An in vivo study of pedicle screw fixation augmenting posterior lumbar interbody fusion. Spine (Phila Pa 1976), 25:858-864. doi: 10.1097/00007632-20000401000015.

7. El Saman A, Meier S, Sander A, Kelm A, Marzi I, Laurer H (2013) Reduced loosening rate and loss of correction following posterior stabilization with or without PMMA augmentation of pedicle screws in vertebral fractures in the elderly. Eur J Trauma Emerg Surg, 39:455-460. doi: 10.1007/s00068-0130310-6.

8. Wilke HJ, Kaiser D, Volkheimer D, Hackenbroch C, Püschel K, Rauschmann M (2016) A pedicle screw system and a lamina hook system provide similar primary and long-term stability: a biomechanical in vitro study with quasi-static and dynamic loading conditions. Eur Spine J, 25:2919-2928. doi: 10.1007/s00586-016-4679-x.

9. Weiser L, Huber G, Sellenschloh K, Viezens L, Püschel K, Morlock MM, Lehmann W (2017) Insufficient stability of pedicle screws in osteoporotic vertebrae: biomechanical correlation of bone mineral density and pedicle screw fixation strength. Eur Spine J, 26: 2891-2897. doi: 10.1007/s00586-0175091-x.

10. Wittenberg RH, Shea M, Swartz DE, Lee KS, White AA, Hayes WC (1991) Importance of bone mineral density in instrumented spine fusions. Spine (Phila Pa 1976), 16: 647-652. doi: 10.1097/00007632199106000-00009.

11. Marc R, Klaus S, Daniel G, Rolf S, Joern WP, Peer E, Karl SD (2010) Mid-range outcomes in 64 consecutive cases of multilevel fusion for degenerative diseases of the lumbar spine. Orthop Rev (Pavia), 2:e3. doi: 10.4081/or.2010.e3.

12. Kim JB, Park SW, Lee YS, Nam TK, Park YS, Kim YB (2015) The effects of spinopelvic parameters and paraspinal muscle degeneration on S1 screw loosening. J Korean Neurosurg Soc,58: 357-362. doi: $10.3340 / j k n s .2015 .58 .4 .357$.

13. A G Brantley, J K Mayfield, J B Koeneman, K R Clark (1994) The effects of pedicle screw fit. An in vitro study. Spine (Phila Pa 1976), 19:1752-1758. doi: 10.1097/00007632- 199408000-00016.

14. Krag MH (1991) Biomechanics of thoracolumbar spinal fixation: A review. Spine (Phila Pa 1976),16:S84-99. doi: 10.1097/00007632-199103001-00014.

15. Krag MH, Beynnon BD, Pope MH, Frymoyer JW, Haugh LD, Weaver DL (1986) An internal fixator for posterior application to short segments of the thoracic, lumbar, or lumbosacral spine: Design and testing. Clin Orthop, 203:75-98. PMID: 7991058

16. Ko CC, Tsai HW, Huang WC, Wu JC, Chen YC, Shih YH, Chen HC, Wu CL, Cheng H (2010) Screw loosening in the Dynesys stabilization system: radiographic evidence and effect on outcomes. Neurosurg Focus, 28:E10. doi: 10.3171/2010.3.FOCUS1052. 
17. Dakhil-Jerew F, Jadeja H, Cohen A, Shepperd JA (2009) Inter-observer reliability of detecting Dynesys pedicle screw using plain X-rays: a study on 50 postoperative patients. Eur Spine J, 18:1486-1493. doi: 10.1007/s00586-009-1071-0.

18. Schreiber JJ, Anderson PA, Rosas HG (2011) Hounsfield units for assessing bone mineral density and strength : a tool for osteoporosis management. J Bone Joint Surg Am, 93:1057-1063. doi: 10.2106/JBJS.J.00160.

19. Sanden B, Olerud C, Petren-Mallmin M, Johansson C, Larsson S (2004) The significance of radiolucent zones surrounding pedicle screws. Definition of screw loosening in spinal instrumentation. J Bone Joint Surg Br, 86:457-461. doi: 10.1302/0301-620x.86b3.14323.

20. Abul-Kasim K, Ohlin A, Strombeck A, Maly P, Sundgren PC (2010) Radiological and clinical outcome of screw placement in adolescent idiopathic scoliosis: evaluation with low-dose computed tomography. Eur Spine J, 19:96-104. doi: 10.1007/s00586-009-1203-6.

21. Abul-Kasim K, Strombeck A, Ohlin A, Maly P, Sundgren PC (2009) Reliability of low-radiation dose CT in the assessment of screw placement after posterior scoliosis surgery, evaluated with a new grading system. Spine (Phila Pa 1976), 34:941-948. doi: 10.1097/BRS.0b013e31819b22a4.

22. Xiaoliang W, Jiawei S, Jinyan W, Yongquan C, Kaiwen P, Jianting C, Hui J (2019) Pedicle screw loosening: the value of radiological imagings and the identification of risk factors assessed by extraction torque during screw removal surgery. Orthop Surg Res,14:6. doi: 10.1186/s13018-0181046-0.

23. Watkins MB (1953) Posterolateral fusion of the lumbar and lumbosacral spine. J Bone Joint Surg Am, 35:1014-1019. PMID: 13108907

24. Tsirikos Al, Sud A, McGurk SM. Radiographic and functional outcome of posterolateral lumbosacral fusion for low grade isthmic spondylolisthesis in children and adolescents. Bone Joint J, 98:88-96. doi: 10.1302/0301-620X.98B1.35672.

25. Pradhan BB, Nassar JA, Delamarter RB (2002) Single-level lumbar spine fusion: a comparison of anterior and posterior approaches. J Spinal Disord Tech,15:355-361. doi: 10.1097/00024720200210000-00003.

26. Ghogawala Z, Benzel EC, Amin-Hanjani S (2004) Prospective outcomes evaluation after decompression with or without instrumented fusion for lumbar stenosis and degenerative Grade I spondylolisthesis. J Neurosurg (Spine), 1:267-272. doi: 10.3171/spi.2004.1.3.0267.

27. Dickman CA, Fessler RG, MacMillan M (1992) Transpedicular screw-rod fixation of the lumbar spine: operative technique and outcome in 104 cases. J Neurosurg, 77:860-870. doi: 10.3171/jns.1992.77.6.0860.

28. Kim DH, Hwang RW, Lee GH, Joshi R, Baker KC, Arnold P, Sasso R, Park D, Fischgrund J (2020) Comparing rates of early pedicle screw loosening in posterolateral lumbar fusion with and without transforaminal lumbar interbody fusion. Spine J, 20:1438-1445. doi: 10.1016/j.spinee.2020.04.021.

29. Macki M, Bydon M, Weingart R (2015) Posterolateral fusion with interbody for lumbar spondylolisthesis is associated with less repeat surgery than posterolateral fusion alone. Clin Neurol 
Neurosurg, 138:117-123. doi: 10.1016/j.clineuro.2015.08.014.

30. Yijian Z, Hao L, Huilin Y, Bin P (2018) Comparison of posterolateral fusion and posterior lumbar interbody fusion for treatment of degenerative spondylolisthesis: Analysis of spino-pelvic sagittal balance and postoperative chronic low back pain. Clin Neurol Neurosurg, 171: 1-5. doi:

10.1016/j.clineuro.2018.04.020.

31. Halvorson TL, Kelley LA, Thomas KA (1994) Effects of bone mineral density on pedicle screw fixation. Spine (Phila Pa 1976), 21:2415-2420. doi: 10.1097/00007632-199411000- 00008.

32. Choi MK, Kim SM, Lim JK (2016) Diagnostic efficacy of Hounsfield units in spine CT for the assessment of real bone mineral density of degenerative spine: correlation study between T-scores determined by DEXA scan and Hounsfield units from CT. Acta Neurochir (Wien), 158: 1421-1427. doi: 10.1007/s00701-016-2821-5.

33. Ebbesen EN, Thomsen JS, Beck-Nielsen H (1999) Lumbar vertebral body compressive strength evaluated by dual-energy X-ray absorptiometry, quantitative computed tomography, and ashing. Bone, 25: 713-724. doi: 10.1016/s8756-3282(99)00216-1.

34. Zou D, Jiang S, Zhou S (2020) Prevalence of osteoporosis in patients undergoing lumbar fusion for lumbar degenerative diseases: a combination of DXA and Hounsfield units. Spine (Phila Pa 1976), 45: E406-E410. doi: 10.1097/BRS.0000000000003284.

35. Lee S, Chung CK, Oh SH (2013) Correlation between bone mineral density measured by Dual-Energy X-Ray absorptiometry and Hounsfield Units measured by diagnostic CT in lumbar spine. J Korean Neurosurg Soc,54:384-389. doi: 10.3340/jkns.2013.54.5.384.

36. Pickhardt PJ, Pooler BD, Lauder T (2013) Opportunistic screening for osteoporosis using abdominal computed tomography scans obtained for other indications. Ann Intern Med, 158:588-595. doi: 10.7326/0003-4819-158-8-201304160-00003.

37. Lee SJ, Binkley N, Lubner MG (2015) Opportunistic screening for osteoporosis using the sagittal reconstruction from routine abdominal CT for combined assessment of vertebral fractures and density. Osteoporosis Int, 27:1131-1136. doi: 10.1007/s00198-015-3318-4.

38. Hendrickson NR, Pickhardt PJ, Del Rio AM (2018) Bone mineral density T-Scores derived from CT attenuation numbers (Hounsfield Units): Clinical utility and correlation with Dual-energy X-ray absorptiometry. Iowa Orthop J, 38:25-31.

39. Jan Bredow, C K Boese, C M L Werner, J Siewe, L Löhrer, K Zarghooni, P Eysel, M J Scheyerer (2016) Predictive validity of preoperative CT scans and the risk of pedicle screw loosening in spinal surgery. Arch Orthop Trauma Surg, 136:1063-1067. doi: 10.1007/s00402-016-2487-8.

40. Burval DJ, McLain RF, Milks R, Inceoglu S (2007) Primary pedicle screw augmentation in osteoporotic lumbar vertebrae: biomechanical analysis of pedicle fixation strength. Spine (Phila Pa 1976), 32:1077-1083. doi: 10.1097/01.brs.0000261566.38422.40.

41. Chen YL, Chen WC, Chou CW, Chen JW, Chang CM, Lai YS, Cheng CK, Wang ST (2014) Biomechanical study of expandable pedicle screw fixation in severe osteoporotic bone comparing 
with conventional and cement-augmented pedicle screws. Med Eng Phys, 36:1416-1420. doi: 10.1016/j.medengphy.2014.05.003.

42. Wu ZX, Gong FT, Liu L (2012) A comparative study on screw loosening in osteoporotic lumbar spine fusion between expandable and conventional pedicle screws. Arch Orthop Trauma Surg,132:471476. doi: 10.1007/s00402-011-1439-6.

43. Xie Y, Fu Q, Chen ZQ, Shi ZC (2011) Comparison between two pedicle screw augmentation instrumentations in adult degenerative scoliosis with osteoporosis. BMC Musculoskelet Disord, 12: 286. doi: 10.1186/1471-2474-12-286.

44. Obeid I, Berjano P, Lamartina C (2019) Classification of coronal imbalance in adult scoliosis and spine deformity: a treatment-oriented guideline. Eur Spine J, 28:94-113. doi: 10.1007/s00586-0185826-3.

45. Bao H, Zhu F, Liu Z (2014) Coronal curvature and spinal imbalance in degenerative lumbar scoliosis: disc degeneration is associated. Spine (Phila Pa 1976), 39:E1441-E1447. doi: 10.1097/BRS.0000000000000603.

46. Liu Z, Zhao ZH, Hu AS (2017) Lumbosacral levelization during surgery reduce the risk of postoperative coronal imbalance in degenerative lumbar scoliosis after long spinal fusion. Chin $\mathrm{J}$ Orthop, 37:193-200.

47. Zou F, Lv F, Xia X (2019) Coronal imbalance correction with distraction of distal convex side of lumbar scoliosis in degenerative lumbar scoliosis. Chin J Orthop, 39: 975-981.

48. Wang H, Zou D, Sun Z, Wang L, Ding W, Li W (2020) Hounsfield Unit for assessing vertebral bone quality and asymmetrical vertebral degeneration in degenerative lumbar scoliosis. Spine (Phila Pa 1976), 45:1559-1566. doi: 10.1097/BRS.0000000000003639.

49. Wang H, Wang L, Sun Z, Jiang S, Li W (2020) Posterior column osteotomy plus unilateral cage strutting for correction of lumbosacral fractional curve in degenerative lumbar scoliosis. $\mathrm{J}$ Orthop Surg Res,15:482. doi: 10.1186/s13018-020-02011-y.

\section{Figures}




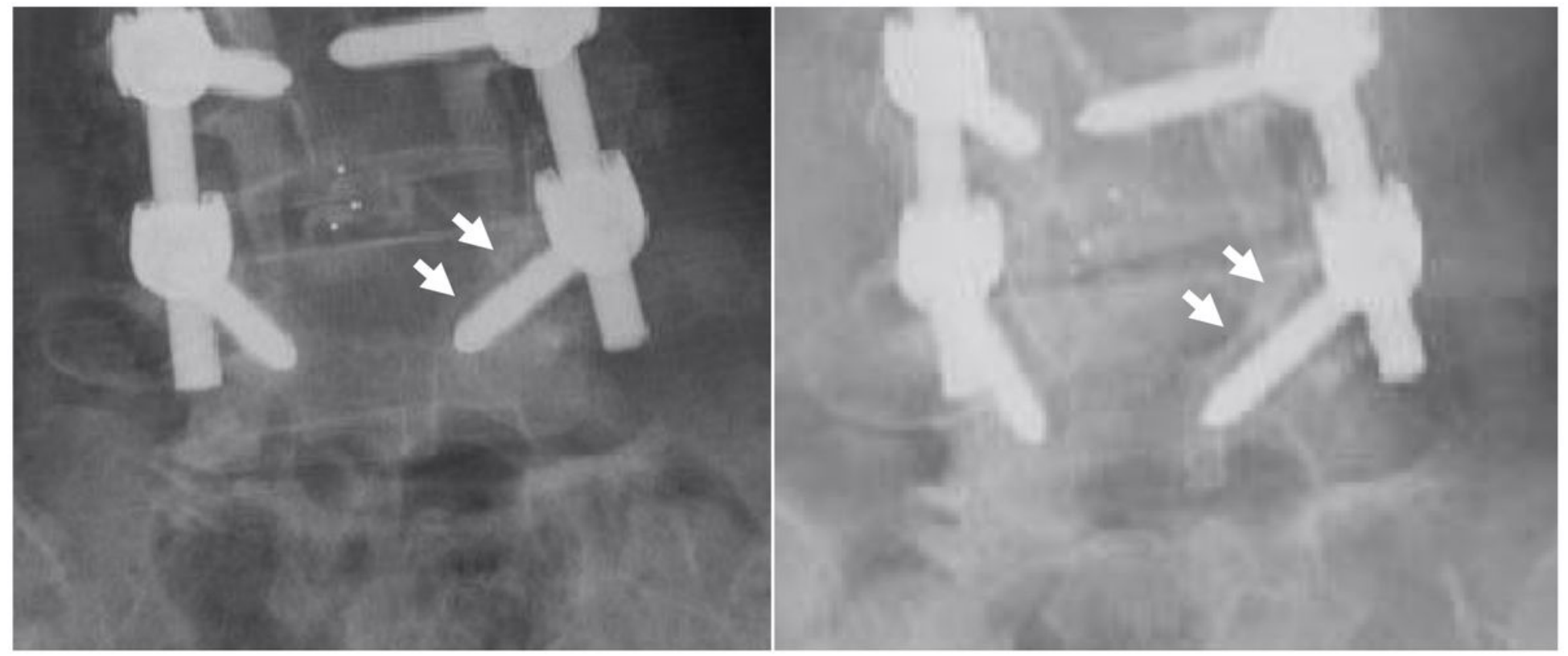

\section{Figure 1}

Left: A halo zone sign (arrows) was defined as radiolucent area (thicker than $1 \mathrm{~mm}$ ) around screw, indicated loosening of the left L5 pedicle screw. Right: A double halo sign (arrows) further confirmed the loosening, described as a radiolucent rim surrounding the screw that is framed by the rim of radiopaque dense bone trabiculae.

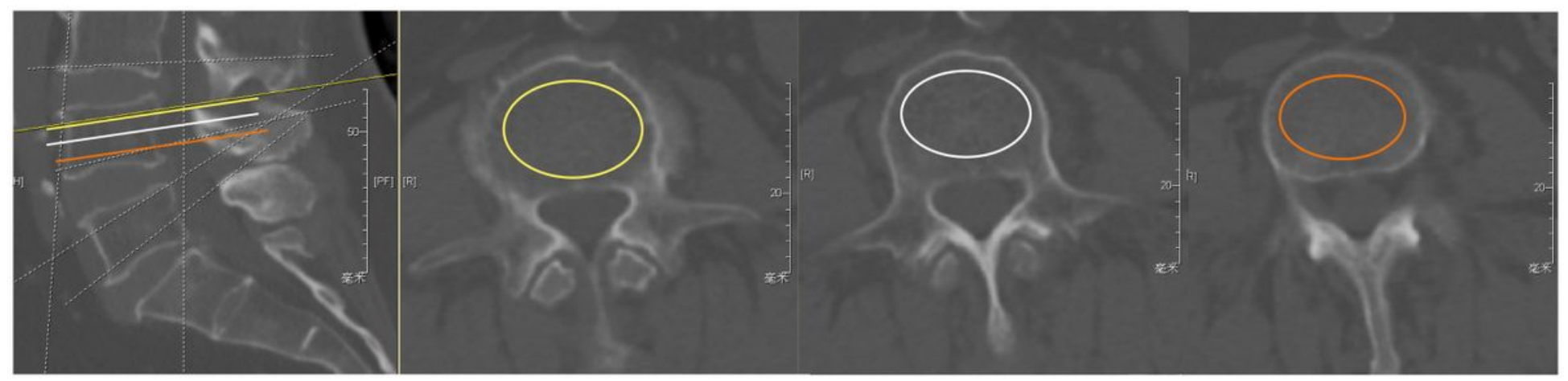

\section{Figure 2}

The trabecular HU measurement was performed on axial images at three levels within the vertebral body: immediately caudal to the superior end plate, mid-body, and cranial to the inferior end plate. For each measurement, the largest possible elliptical region of interest was drawn, excluding the cortical margins to prevent volume averaging. 


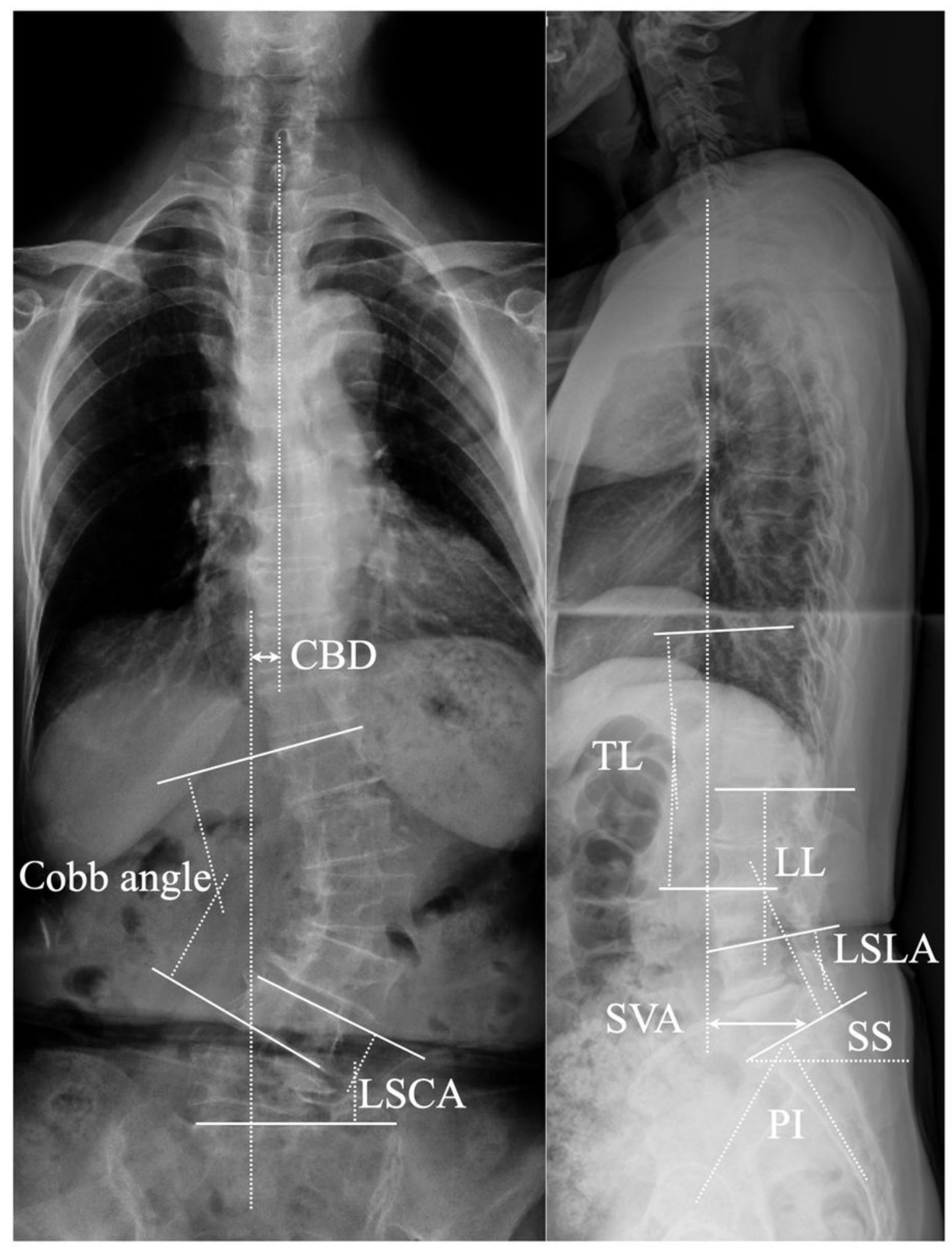

\section{Figure 3}

The radiological data measured on the P/A X-ray include Cobb's angle, lumbosacral coronal angle (LSCA) and coronal balance distance (CBD). The radiological data measured on the P/A X-ray include lumbar lordosis (LL), thoracolumbar junction (TL), pelvic incidence (PI), sacral slope (SS) and sagittal vertical axis (SVA). 

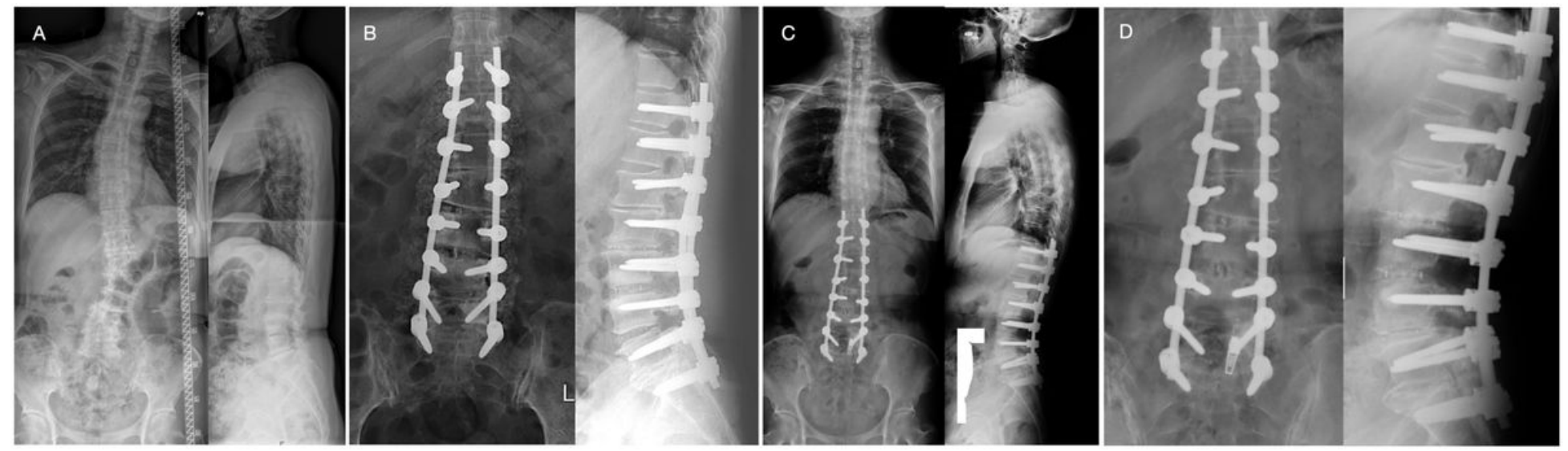

\section{Figure 4}

The patient, male, 63 years old. BMD on L1 vertebrae $=178 \mathrm{HU}$. (A) Preoperative Cobb's angle was 31 degrees, CBD was $47 \mathrm{~mm}$, LSCA was 22 degrees, LL was 26 degrees. (B) He underwent posterior decompression and instrumented fusion (T11-S1) with bilateral posterolateral fusion. At postoperative, Cobb's angle was 8 degrees, LSCA was 7 degrees. (C) At one-year follow up, Cobb's angle was 7 degrees, CBD was $18 \mathrm{~mm}$, LL was 34 degrees. (D) At two-year follow up, posterolateral arthrodesis was satisfactory, and no distal pedicle screw loosening was detected.
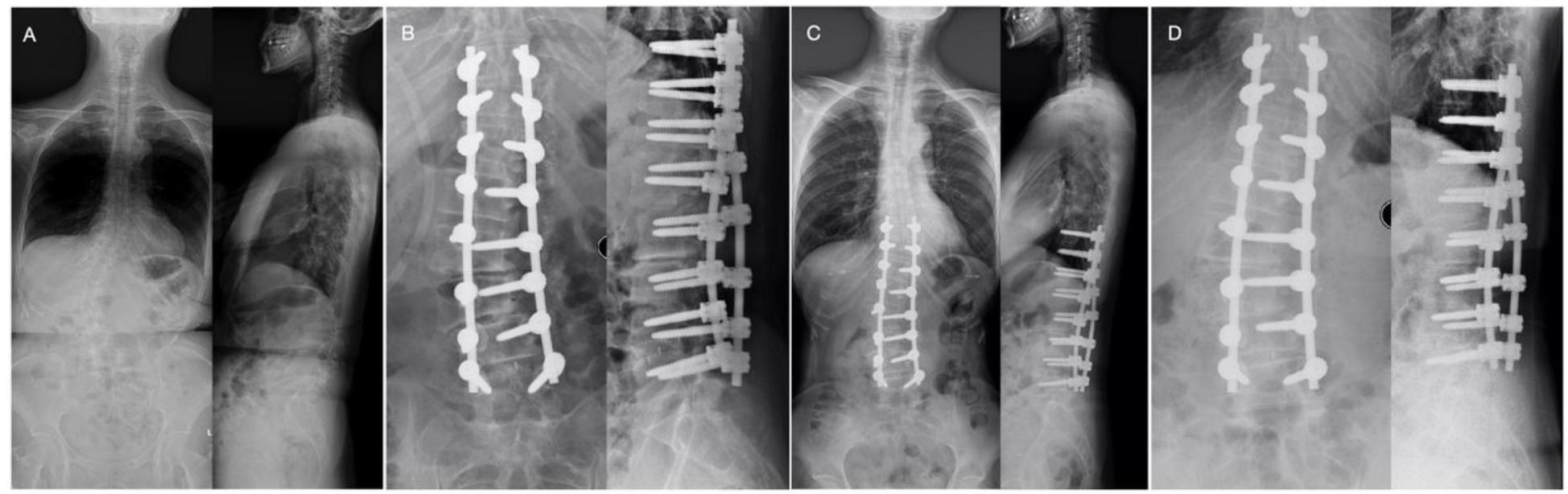

\section{Figure 5}

The patient, female, 63 years old. BMD on L1 vertebrae $=129 \mathrm{HU}$. (A) Preoperative Cobb's angle was 55 degrees, CBD was 23 mm, LSCA was 23 degrees, LL was -14 degrees. (B) He underwent posterior decompression and instrumented fusion (T10-L5) with left posterolateral fusion. At postoperative, Cobb's angle was 22 degrees, LSCA was 7 degrees. (C) At one-year follow up, Cobb's angle was 24 degrees, CBD was $10 \mathrm{~mm}$. (D) At two-year follow up, posterolateral arthrodesis was not satisfactory, bilateral L5 pedicle screw loosening was detected. 